\title{
AUTOMEDICAÇÃO NA PANDEMIA DO NOVO CORONAVÍRUS
}

\author{
SELF-MEDICATION IN THE PANDEMIC OF THE NEW CORONAVIRUS
}

\author{
Alícia de Freitas Silva \\ Jefferson Silva Pinho de Jesus ${ }^{2}$ \\ Juliana Lima Gomes Rodrigues ${ }^{3}$
}

RESUMO: A automedicação consiste em administrar por conta própria um ou mais medicamentos sem a orientação profissional e, com a grande quantidade de informações acessíveis na internet tornou- se um hábito comum no período de pandemia do novo coronavírus. O presente trabalho teve como objetivo expor sobre a utilização inadequada de medicamentos prescritos e isentos devido ao seu fácil acesso durante o isolamento social, retratar a possibilidade de efeitos indesejáveis relacionados aos medicamentos, consequente da automedicação, e destacar a ausência da prática clínica e de assistência nos fornecimentos farmacêuticos ao paciente. Os dados foram obtidos entre agosto de 2020 e maio de 2021. A Cloroquina/hidroxicloroquina, vitamina C, ivermectina, azitromicina, ibuprofeno e lopinavir-ritonavir são os medicamentos mais citados nos artigos científicos. Os antiretrovirais foram relatados em sinergia com cloroquina/hidroxicloroquina, que foi a mais citada nos artigos relacionados à automedicação. $O$ uso incorreto dessas substâncias pode causar efeitos colaterais graves, outros tipos de patologias ou até dependência. Sendo assim, o farmacêutico e a equipe multiprofissional devem orientar o paciente sobre o uso racional de medicamentos.

Palavras-chave: Automedicação. Pandemia. Coronavírus.

ABSTRACT: Self-medication consists of administering one or more medications on your own without professional guidance and, with the large amount of information accessible on the internet, it became a common habit in the pandemic period of the new coronavirus. This study aimed to expose the inappropriate use of prescription and exempt drugs due to its easy

\footnotetext{
I Graduação em farmácia Instituição de ensino: Universidade Salvador (UNIFACS). Email:aliciafs2or7@gmail.com.

2 Graduação em farmácia. Instituição de ensino: Universidade Salvador (UNIFACS) .E-mail: jeff.silvar998@gmail.com.

3 Docente do curso de farmácia. Instituição de ensino: Universidade Salvador (UNIFACS). Email: juliana.rodrigues@unifacs.br.
} 
access during social isolation, to portray the possibility of undesirable effects related to drugs, resulting from self-medication, and to highlight the absence of clinical practice and assistance with pharmaceutical supplies for the patient. Data were obtained between August 2020 and April 2021. Chloroquine/hydroxychloroquine, vitamin C, ivermectin, azithromycin, ibuprofen and lopinavir-ritonavir are the drugs most cited in scientific articles. Antiretrovirals have been reported in synergy with chloroquine / hydroxychloroquine, which was the most cited in articles related to self-medication. The incorrect use of these substances can cause serious side effects, other types of pathologies or even addiction. Therefore, the pharmacist and the multidisciplinary team should advise the patient on the rational use of medications.

Keywords: Self-medication. Pandemic. Coronavírus.

\section{INTRODUÇÃO}

A COVID-ı9 é uma infecção respiratória que causa sintomas similares à gripe, podendo evoluir para uma pneumonia grave, causando cansaço, perda do paladar ou olfato, febre e dificuldade para respirar. Segundo a Organização Mundial de Saúde (OMS) o mundo já ultrapassou a marca de 147 milhões de infectados e mais de 3 milhões de mortos, sendo os Estados Unidos, Índia e Brasil os países mais afetados pela pandemia. Por ser um vírus novo, ainda não há medicamentos específicos para a cura ou contenção dos sintomas da doença, porém já estão sendo feitos estudos clínicos com medicamentos e já foram desenvolvidas algumas vacinas. Com a pandemia do novo coronavírus,diversas medidas têm sido utilizadas com o intuito de diminuir o contágio pelo vírus. $\mathrm{O}$ isolamento social separa as pessoas doentes das não doentes, enquanto o distanciamento social visa a redução de aglomerações de pessoas; já a quarentena é a retenção de atividades e separação de indivíduos que provavelmente foram expostos ao vírus. A população pode optar em não buscar pelo atendimento presencial por anseio de se contaminar, entretanto os casos de automedicação têm aumentado nesse período, o que pode provocar efeitos indesejados e ser nocivo ao usuário (Lei et al., 2018; ONCHONGA, 2020).

Existe também a telemedicina, que é uma iniciativa realizada por meio de tecnologias digitais, possibilitando serviços à distância para o cuidado com a saúde, proporcionando a assistência médica online a pacientes, clínicas, hospitais e profissionais $d a$ saúde (MALDONADO, 2016). Dentro do isolamento social a internet se tornou a maior fonte de informações em saúde para os mais leigos, fontes de pesquisa direcionadas à internet reportam aumento de procura por medicamentos, suplementos e chás, em sites de compra ou bula sugerindo a prática de automedicação e auto dosagem (ONCHONGA, 2020), todavia, os usuários utilizam os ativos para alívio de sintomas ou complicações, alguns sem saber o intervalo de tempo determinado entre duas dosagem em curto período de tempo tendo capacidade provocar efeitos indesejáveis (ONCHONGA, 2020).

Nas farmácias e drogarias há venda de medicamentos em um comércio potencial onde muitos que exigem controle especial são vendidos sem receita e com ausência da 
assistência e orientação ao paciente permitindo livre escolha (MACEDO et al, 2016). De modo que há um afastamento da consulta nos setores de saúde, dificultando a prática clínica do cuidado cuja importância na segurança dos pacientes é imprescindível para adotar medidas na intenção de manter solidificação do sistema, prevenir acidentes associados aos fármacos aumentando a probabilidade de resultados favoráveis e conter mortes que podem ser evitadas dentro do sistema de saúde (SILVA, 2016).

Durante a pandemia do novo coronavírus a população tem se automedicado com o objetivo de tratar ou aliviar sintomas como: febre, tosse, coriza, dores musculares, dores de cabeça e dores de garganta, que se assemelham com os sintomas da doença COVID-19. A importância desse estudo se dá em expor o uso indiscriminado de medicamentos e o seu fácil acesso, exibir os medicamentos mais utilizados e mostrar os seus efeitos indesejáveis. Este trabalho tem como objetivo realizar levantamento bibliográfico sobre a automedicação na pandemia do novo coronavírus, abordando a utilização inadequada de medicamentos prescritos e isentos de prescrição com fácil acesso durante o isolamento social da pandemia.

\section{METODOLOGIA}

O trabalho trata-se de uma revisão bibliográfica, cujo tema é a automedicação na pandemia do novo coronavírus, o problema que direcionou o seu seguimento foi: A Automedicação pode ter a capacidade de desencadear efeitos indesejáveis no isolamento social? Refere-se a uma pesquisa qualitativa e quantitativa, com o intuito de apontar o uso indevido de medicamentos durante o isolamento na pandemia da COVID-I9.

Para auxiliar na procedência do artigo serão apontadas referências com o suporte de dados em sites como: Google Acadêmico, Scientific Electronic Library Online (SCIELO), NCBI (The National Center for Biotechnology Information), PubMed, revistas de saúde e o site da OMS (Organização mundial da saúde), em inglês e português. $O$ artigo foi elaborado entre agosto de 2020 e maio de 202I, sendo consideradas publicações entre os anos 2012 a 202I, fornecendo as informações atualizadas para a elaboração deste artigo. Como critérios de exclusão deste trabalho estão materiais que não apresentam característica, especificidade, dados quantitativos relacionados ao tema e artigos que não apresentam títulos com as palavras-chave usadas neste estudo.

\section{RESULTADOS E DISCUSSÃO}

O artigo foi elaborado em concordância com a metodologia, empregando as palavraschave "automedicação", "pandemia”, "coronavírus", em combinação com "uso excessivo de medicamentos", "uso racional de medicamentos" ou "efeitos colaterais", e suas correspondentes em inglês. A pesquisa teve o Google Acadêmico, NCBI (The National Center for Biotechnology Information) e PubMed como principais fontes de dados, foram vistos aproximadamente 827 resultados de trabalhos que se referem aos objetivos, porém somente 24 artigos foram selecionados por apresentarem dados de grupos de indivíduos de diferentes países relacionados a automedicação na pandemia, outros artigos foram selecionados devido 
aos dados quantitativos mundiais conforme a coesão entre causa da automedicação e a toxicidade dos medicamentos consumidos.

Figura I - Fluxograma de estudo

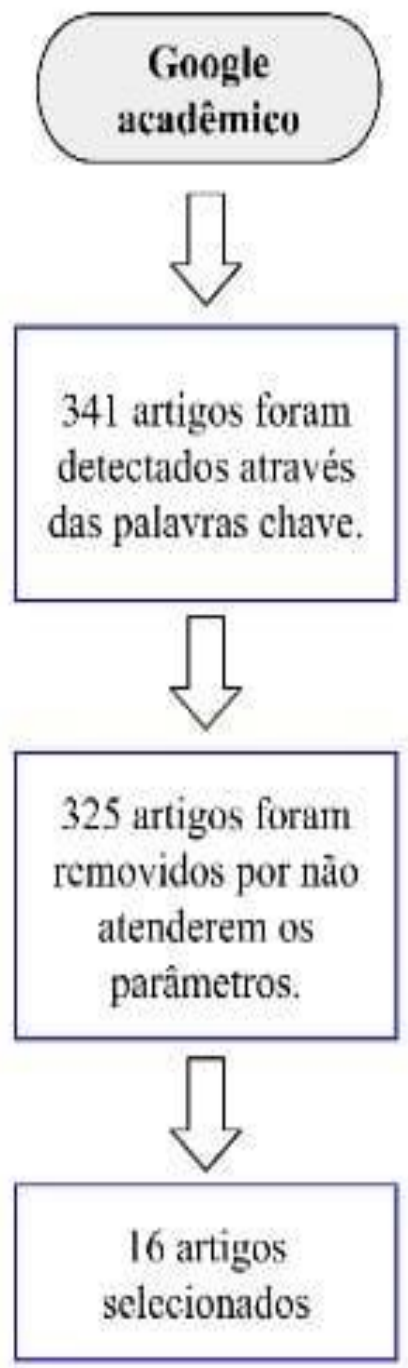

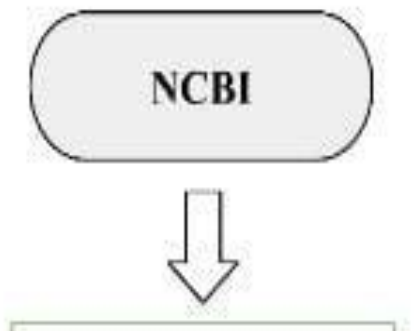

257 artigos foram detectados através das palavras chave.

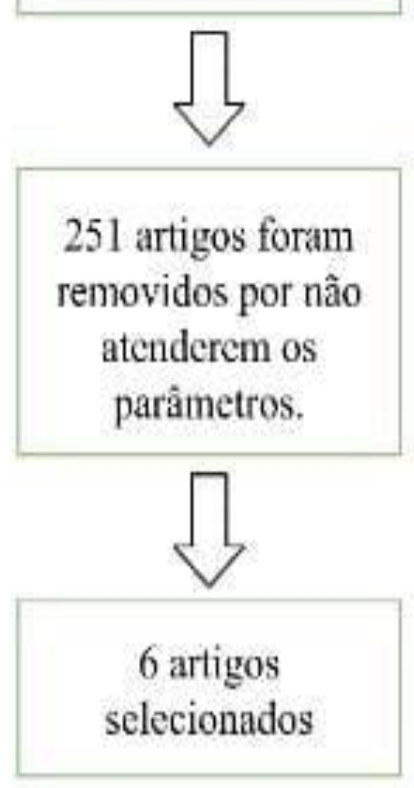

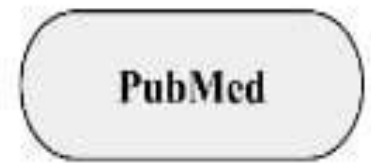

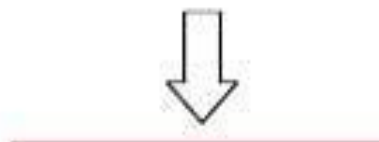

229 artigos foram detectados através das palavras chave.

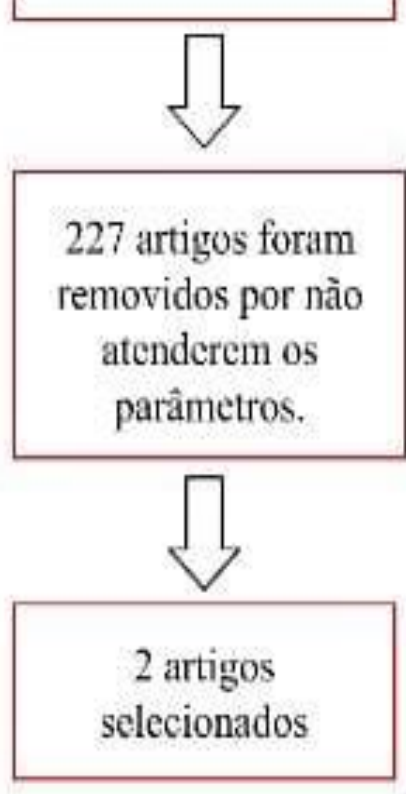

Fonte: Próprio autor

Indivíduos que praticam automedicação e auto dosagem rotineiramente, aderem a prática do uso irracional de medicamentos devido ao seu fácil acesso permitindo a auto administração que pode ser nociva ao usuário. Segundo o Ministério da Saúde, os relaxantes musculares, anti térmicos e os que têm efeito no sistema nervoso estão relacionados com a maior parte das intoxicações. 
Figura 2- Medicamentos mais citados em artigos científicos no período de julho de 2020 a fevereiro de 2021

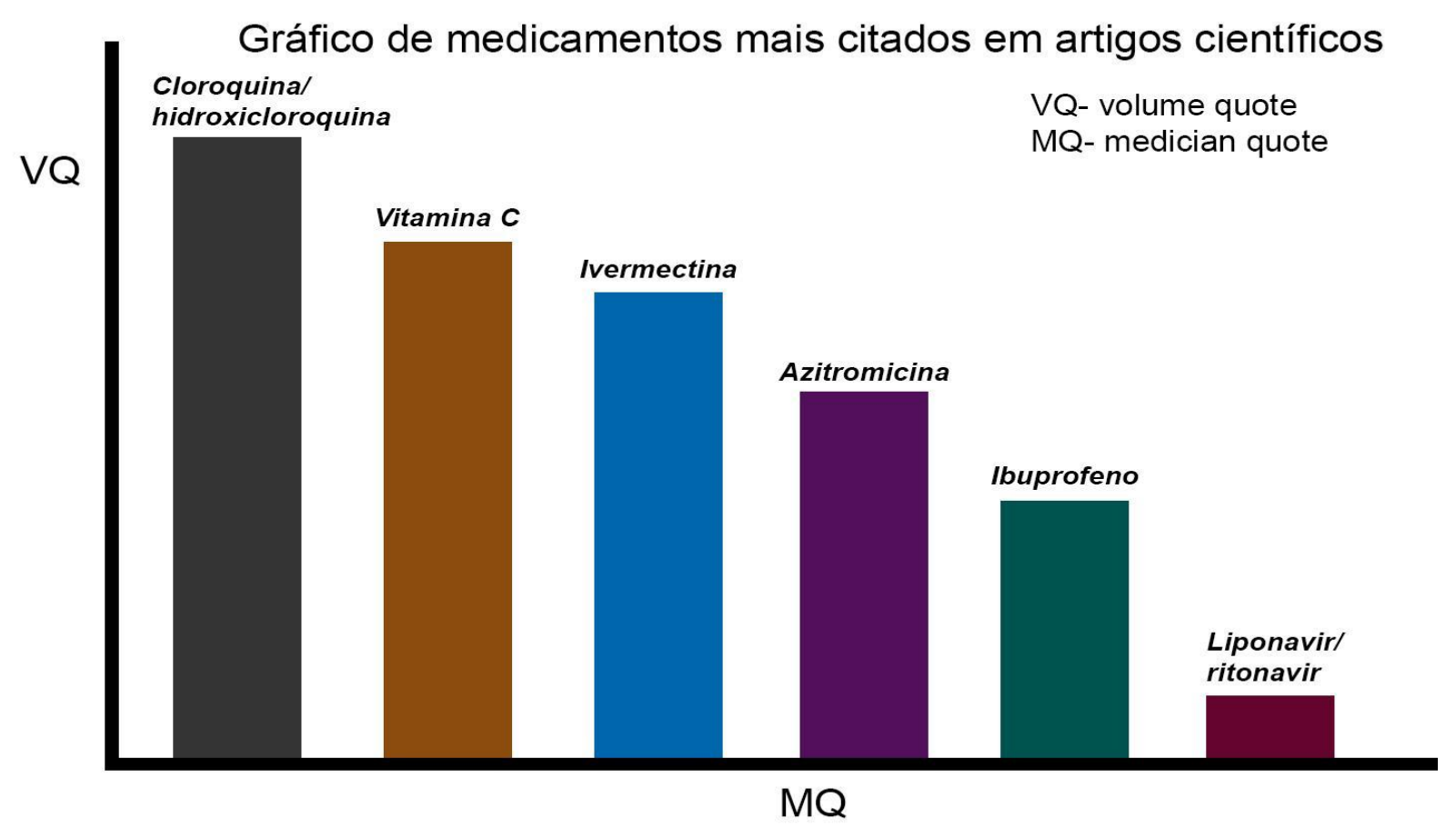

Fonte: Próprio autor

O gráfico salienta os principais medicamentos citados em artigos científicos que se encaixam nos critérios de inclusão, salientando os medicamentos mais utilizados na pandemia, o VQ (volume quote) é relacionado ao volume de citação do medicamento em artigos científicos e o MQ (medician quote) indica quais medicamentos foram citados, contagem realizada pelos artigos selecionados. Hidroxicloroquina/cloroquina foi a mais citada nos artigos selecionados sobre a automedicação e os anti-retrovirais sendo relatados geralmente em sinergia com cloroquina/hidroxicloroquina em alguns artigos científicos (Goudarzi et al., 2020; Matias et al., 2020).

A procura por vitaminas e suplementos também teve grande crescimento. A vitamina $\mathrm{C}$ e a utilização de multi vitaminas/suplementos pode ser muito perigosa, pois tem o potencial de causar hipervitaminose. Alguns estudos descrevem que a vitamina $\mathrm{C}$ causa efeitos adversos semelhantes aos dos medicamentos citados acima, como: náusea, vômito, dores de estômago e dor de cabeça, todavia, não há clareza sobre a interação medicamentosa com a Vitamina C (Goudarzi et al., 2020; Matias et al., 2020).

As principais razões que ocasionaram os consumidores a praticar a automedicação na pandemia foram a prevenção e a melhoria dos sintomas, independentemente de serem positivos ou negativos, evitando o atendimento e a realização do teste (Muhammed J. et all., 2020). Quispe-Canãri et al., 202I) aponta que os analgésicos, antibióticos, antifúngicos e anti 
oxidantes são os mais procurados, como por exemplo o paracetamol, azitromicina, ibuprofeno, anti-retrovirais, cloroquina e hidroxicloroquina, penicilina, dipirona, ivermectina e vitamina $\mathrm{C}$.

Estudos transversais demonstram a prática do autocuidado em diversos países, entretanto, é variável a sua fração de praticantes tendo uma margem de $32 \%$ a $85 \%$ em países como: Sérvia, Montenegro, Japão, Índia, China, Brasil, Ásia e Etiópia (Muhammed J. et all, 2020; Aster, 2018). Em destaque na gama de medicamentos, a vitamina $\mathrm{C}$ é a mais consumida pela comunidade na tentativa de prevenir a contaminação ou fortalecer a imunidade. Estudos apontam que 32\% do total dos indivíduos dos países citados planejam ou já se automedicaram (Alvarez-Risco et al., 2020; Arnold J et al., 2021; Tasnim et al., 2020).

Já a cloroquina e a hidroxicloroquina foram as mais descritas no ambiente intrahospitalar em uso off-label, pois devido a sua exigência de prescrição médica é mais difícil sua aplicação no âmbito doméstico. São medicamentos usados no tratamento de malária e lúpus, porém teve a possível indicação para a cura do COVID-19 pelo fato de ter ocorrido estudos in vitro para testar a eficácia como antiviral em 2003, quando houve a epidemia da SARS-CoV-I. Houve efeito na replicação viral, mas como a epidemia não chegou a se espalhar os testes foram interrompidos e agora na pandemia do novo coronavírus foram resgatados, contudo, não há indicação comprovada e com a falta de um medicamento com a eficácia determinada para a cura do COVID-i9 as pessoas continuaram se automedicando (RODRIGUES, 2020). Maioritariamente possui cardiotoxicidade, prolongando o intervalo QT por inibição da $\mathrm{CYP}_{2} \mathrm{D} 6$ e do transportador $\mathrm{ABCB} / \mathrm{Pgp}$ que pertencem a um grupo de proteínas responsáveis pela metabolização de $25 \%$ das drogas clínicas, facilitando sua eliminação e transporte dessas substâncias (Bertilsson L, 2012). A azitromicina também provoca alteração no intervalo QT, em sinergia com cloroquina/hidroxicloroquina, com o objetivo de diminuir a carga viral, mas alguns estudos contrariam sua eficácia na inibição do vírus, sendo não recomendada (Ágnes Telbisz et al 2020; Alvarez-Risco et al., 2020).

A ivermectina apesar de ter mostrado eficácia contra o vírus apenas em testes in vitro, apresenta atividade inibidora dos sítios de ligação do vírus, com isso o uso indiscriminado está ligado a muitos casos de resistência microbiana, principalmente bacteriana e parasitária, uma pequena fração da concentração da ivermectina tem potencial neurotóxico juntamente com sua atividade hepatotóxica por induzir necrose hepática, sendo ocasionalmente administrado aos pacientes com cloroquina, hidroxicloroquina e azitromicina (Ágnes Telbisz et al., 2020).

Segundo o NIH (National Institutes of Health), um site oficial do governo dos Estados Unidos, relata a ineficácia e toxicidade em alguns casos na emergência da sinergia destes fármacos. Os principais problemas são: hipoglicemia, miopatias, rabdomiólise, mioglobinúria, bloqueio cardíaco ou atraso no intervalo QT e neurotoxicidade, sendo não aprovados para infecções virais. Devido a propagação de informações tendenciosas muitos cidadãos de países diferentes no mundo adotaram a autoadministração da ivermectina com intuito de evitar a infecção pelo Sars-COV 2 (Ágnes Telbisz et al., 2020).

Lopinavir-ritonavir, medicamento usado em ambiente hospitalar que pertence à classe dos inibidores de protease, foi aplicado em alguns pacientes e não demonstrou 
diferença potencial no fator de virulência em comparação aos que não receberam as dosagens do fármaco. A mortalidade com os respectivos antirretrovirais não referiu alteração significativa, tendo os distúrbios gastrointestinais como efeito adverso mais comum, sendo não sugerido para os casos leves, moderados ou graves, impossibilitando o seu uso com os antipiréticos, que são tóxicos no aparelho gastrointestinal (Yanping et al., 2020).

Em situação de isolamento social há chance de alteração no comportamento dos indivíduos, sendo potencialmente praticante de auto dosagem (decisão individual do sujeito de determinar a dose a ser administrada e alterná-las em um intervalo de tempo, com probabilidade de uma superdosagem ou subdosagem), somada a automedicação, que é o ato de administrar um medicamento por conta própria, sem orientação profissional, ampliando as chances de efeitos tóxicos (Marcelo Beltrão, 2020). Isso se deve à ausência da consulta clínica, num cenário onde a internet se tornou centro de informação de saúde e nas farmácias ou drogarias medicamentos são vendidos abundantemente sem a orientação farmacêutica sobre seus efeitos indesejáveis, inviabilizando a prática clínica da assistência em saúde. $\mathrm{O}$ Conselho Nacional de Saúde fdiz que o uso indiscriminado de medicamentos é um problema antigo, de acordo o Ministério da Saúde esta prática é responsável por $27 \%$ das intoxicações. Sendo assim, o Ministério da Saúde defende a utilização de medicamentos de forma segura aos usuários, através da orientação e atenção farmacêutica que é direcionada ao paciente com o objetivo de promover o uso racional dos medicamentos para obter os efeitos esperados (SOBRAL, 2018).

\section{CONSIDERAÇÕES FINAIS}

A automedicação pode trazer efeitos indesejáveis à saúde, porém é uma adversidade global que é praticada há muitos anos antes da pandemia. No isolamento uma boa parte da sociedade utilizou o conhecimento popular através da internet, com informações tendenciosas para se inclinar ao autocuidado independente dos conhecimentos em saúde ou auxílio de um profissional capacitado. Esta ocasião reduziu a busca pelo atendimento hospitalar, visto que, o fácil acesso aos medicamentos concede o poder de decidir realizar a automedicação e autoadministração. É imprescindível que o farmacêutico juntamente com a equipe multiprofissional orientem os pacientes sobre o uso racional de medicamentos e substâncias potencialmente tóxicas, mostrando a possibilidade de causar efeitos nocivos caso as substâncias sejam usadas incorretamente.

\section{REFERÊNCIAS}

ABOBAKER A, Alzwi A, Alraied AHA. Overview of the possible role of vitamin C in management of COVID-19. Pharmacol Rep. 2020 Dec;72(6):1517-1528. doi: 10.1007/s43440o20-oor76-I. Epub 2020 Oct 28. PMID: 33113146; PMCID: PMC7592143. Disponível em: 〈https://pubmed.ncbi.nlm.nih.gov/33113146/〉. Acesso em I5 de abril de 2021. 
CAO, Bin et al. A trial of lopinavir-ritonavir in adults hospitalized with severe Covid-I9. New England Journal of Medicine, 2020. Disponível em: 〈https://www.nejm.org/doi/full/ı.1056/NEJMoa20o1282〉. Acesso em I9 de abril de 2021.

FREITAS, André Ricardo Ribas; NAPIMOGA, Marcelo; DONALISIO, Maria Rita. Análise da gravidade da pandemia de Covid-19. Epidemiologia e Serviços de Saúde, v. 29, p. e2020119, 2020. Disponível em: 〈https://www.scielosp.org/article/ress/2020.v29n2/e2020119/〉. Acesso em 03 de novembro de 2020 .

GÉRARD A, Romani S, Fresse A, Viard D, Parassol N, Granvuillemin A, Chouchana L, Rocher F, Drici MD; French Network of Pharmacovigilance Centers. "Off-label" use of hydroxychloroquine, azithromycin, lopinavir-ritonavir and chloroquine in COVID-I9: A survey of cardiac adverse drug reactions by the French Network of Pharmacovigilance Centers. Therapie. 2020 Jul-Aug;75(4):371-379. doi: 10.1016/j.therap.2020.05.002. Epub 2020 May 7. PMID: 32418730; PMCID: PMC7204701. Disponível em: <https://pubmed.ncbi.nlm.nih.gov/32418730/>. Acesso em Is de abril de 2021.

GOUDARZI, Sogand et al. Effect of Vitamins and Dietary Supplements on Cardiovascular Health. Critical pathways in cardiology, v. 19, n. 3, p. 153-159, 2020. Disponível em: 〈https://www.ingentaconnect.com/content/wk/hpc/2020/oooooor9/ooooooo3/artoooo9〉. Acesso em i6 de abril de 2021.

KASSIE, Aster Desalew et al. "Self-medication practice and associated factors among adult household members in Meket district, Northeast Ethiopia, 2017." BMC pharmacology or toxicology vol. I9,I I5. IO Apr. 2018. Disponível em: 〈https://www.ncbi.nlm.nih.gov/pmc/articles/PMC5894137/>. Acesso em 24 de abril de 2021.

LEI, Xiaosheng et al. Self-medication practice and associated factors among residents in Wuhan, China. International journal of environmental research and public health, v. $15, \mathrm{n}$. I, p. 68, 2018. Disponível em: 〈https://www.mdpi.com/166o-46oI/I5/I/68〉. Acesso em I8 de outubro de 2020 .

MACEDO, Giani Rambaldi et al. O poder do marketing no consumo excessivo de medicamentos no Brasil. Revista Transformar, v. 9, p. II4-I28, 2016. Disponível em: $\langle$ http://www.fsj.edu.br/transformar/index.php/transformar/article/view/79〉. Acesso em 27 de fevereiro de 202I.

MALDONADO, Jose Manuel Santos de Varge; MARQUES, Alexandre Barbosa; CRUZ, Antonio. Telemedicina: desafios à sua difusão no Brasil. Cadernos de Saúde Pública, v. 32, 
p. eoor5s6i5, 2016. Disponível em: $\langle$ https://www.scielosp.org/article/csp/2016.v32suppl2/eoor55615/pt/>. Acesso em $30 \mathrm{de}$ abril de 2021.

MALIK, Muna et al. "Self-medication during Covid-I9 pandemic: challenges and opportunities." Drugs d therapy perspectives : for rational drug selection and use, 1-3. 3 Oct. 2020. Disponível em: 〈https://www.ncbi.nlm.nih.gov/pmc/articles/PMC7532737/>. Acesso em 24 de abril de 202I.

MALIK M, Tahir MJ, Jabbar R, Ahmed A, Hussain R. Automedicação durante a pandemia de Covid-rg: desafios e oportunidades [publicado online antes da impressão, em 3 de outubro de 2020]. Drugs Ther Perspect . 2020; I-3. doi: 10.1007 / s40267-020-00785-z. Disponível em: 〈https://www.ncbi.nlm.nih.gov/pmc/articles/PMC7532737/>. Acesso em i9 de março de 2021.

MOLENTO, Marcelo Beltrão. COVID-ig and the rush for self-medication and self-dosing with ivermectin: A word of caution. One Health, v. Io, p. Ioor48, 2020. Disponível em: 〈https://www.ncbi.nlm.nih.gov/pmc/articles/PMC7313521/〉. Acesso em I9 de março de 2021.

ONCHONGA, David. A Google Trends study on the interest in self-medication during the 2019 novel coronavirus (COVID-19) disease pandemic. Saudi Pharmaceutical Journal: 946 SPJ, v. 28, n. 7, p. 903, 2020. Disponível em: 〈https://www.ncbi.nlm.nih.gov/pmc/articles/PMC $7299852 /$. Acesso em 28 de outubro de 2020 .

ONCHONGA, David et al. Prevalence of fear of childbirth in a sample of gravida women in Kenya. Sexual \& Reproductive Healthcare, p. Iо0510, 2020. Disponível em: 〈https://www.sciencedirect.com/science/article/pii/Si877575620300227〉. Acesso em 2i de outubro de 2020 .

ONCHONGA, David et al. Women 's experience from midwife-led, integrated pre-birth training; and its influence on childbirth fear. A qualitative interview study. Sexual \& Reproductive Healthcare, p. I00512, 2020. Disponível em: 〈https://www.sciencedirect.com/science/article/pii/Si87757562030135X〉. Acesso em i9 de novembro de 2020 .

QUISPE-CAÑARI, Jean Franco et al. Práticas de automedicação durante a pandemia de COVID-Ig entre a população adulta no Peru: um estudo transversal. Saudi Pharmaceutical Journal , v. 29, n. I, pág. I-II, 202I. Disponível em: 〈https://www.sciencedirect.com/science/article/pii/Si319016420302760〉. Acesso em i9 de março de 202I. 
RODRIGUES, Léo Peixoto. A controvérsia científica em torno da cloroquina e hidroxicloroquina no tratamento da Covid-I9: a importância dos estudos sociais da ciência na sociedade complexa. Simbiótica. Revista Eletrônica, v. 7, n. I, p. 147-171, 2020. Disponível em: 〈https://periodicos.ufes.br/simbiotica/article/view/30988〉. Acesso em os de maio de 2021.

SADIO, AJ, Gbeasor-Komlanvi, FA, Konu, RY et al. Avaliação das práticas de automedicação no contexto do surto de COVID-I9 no Togo. BMC Public Health 21, 58 (2021). https://doi.org/Io.I186/si2889-020-I0145-I. Disponível em: 〈https://bmcpublichealth.biomedcentral.com/articles/ıo.II86/sı2889-020-IoI45-I\#citeas〉. Acesso em i9 de março de 202I.

SILVA, Carla Yasmin Alves Batista; PONCIANO, Anna Karollayne Bezerra; LUZ, Dayse Christina Rodrigues Pereira. AUTOMEDICAÇÃO DURANTE A PANDEMIA DA COVID-I9. Revista E-Ciência, v. 8, n. 2, 2021. Disponível em: $\langle$ http://www.revistafjn.com.br/revista/index.php/eciencia/article/view/967〉. Acesso em I9 de março de 202I.

SILVA, Luziane Teixeira de Castro. Análise da automedicação, suas práticas e riscos sobre a saúde: revisão de literatura. 2016. Disponível em: 〈http://131.0.244.66:8082/jspui/handle/123456789/150〉.Acesso em 27 de fevereiro de 2021.

SOBRAL, Camila C. et al. A importância do uso racional de medicamentos. FACIDERRevista Científica, n. II, 2018. Disponível em: <http://revista.seicesucol.edu.br/index.php/facider/article/view/r67>. Acesso em 27 de fevereiro de 2021.

TEH LK, Bertilsson L (2012). "Pharmacogenomics of CYP 2 D6: molecular genetics, interethnic differences and clinical importance". Drug Metabolism and Pharmacokinetics. 27 (I): 55-67. Disponível em: 〈https://doi.org/ro.2133\%2Fdmpk.DMPK-II-RV-I2I >. Acesso em 24 de abril de 2021.

TELBISZ, Agnes et al. Interactions of anti-COVID-I9 drug candidates with multispecific $\mathrm{ABC}$ and OATP drug transporters. bioRxiv, 2020. Disponível em: 〈https://www.biorxiv.org/content/Io.IIOI/2020.11.21.392555Vi.full〉. Acesso em I9 de março de 202I.

TUMELERO, N. Pesquisa exploratória: conceito, características e aplicação em 4 passos. conceito, características e aplicação em 4 passos. Blog Mettzer [03/04/2019] v. 22, n. 05, 2020. Disponível

em: 〈https://blog.mettzer.com/pesquisa-exploratoria/>. Acesso em 27 de fevereiro de 2021. 